\title{
Ground state alternative for $p$-Laplacian with potential term
}

\author{
Yehuda Pinchover \\ Department of Mathematics \\ Technion - Israel Institute of Technology \\ Haifa 32000, Israel \\ pincho@techunix.technion.ac.il
}

\author{
Kyril Tintarev \\ Department of Mathematics \\ Uppsala University \\ SE-751 06 Uppsala, Sweden \\ kyril.tintarev@math.uu.se
}

July 9, 2018

\begin{abstract}
Let $\Omega$ be a domain in $\mathbb{R}^{d}, d \geq 2$, and $1<p<\infty$. Fix $V \in L_{\mathrm{loc}}^{\infty}(\Omega)$. Consider the functional $Q$ and its Gâteaux derivative $Q^{\prime}$ given by $Q(u):=\int_{\Omega}\left(|\nabla u|^{p}+V|u|^{p}\right) \mathrm{d} x, \quad \frac{1}{p} Q^{\prime}(u):=-\nabla \cdot\left(|\nabla u|^{p-2} \nabla u\right)+V|u|^{p-2} u$. If $Q \geq 0$ on $C_{0}^{\infty}(\Omega)$, then either there is a positive continuous function $W$ such that $\int W|u|^{p} \mathrm{~d} x \leq Q(u)$ for all $u \in C_{0}^{\infty}(\Omega)$, or there is a sequence $u_{k} \in C_{0}^{\infty}(\Omega)$ and a function $v>0$ satisfying $Q^{\prime}(v)=0$, such that $Q\left(u_{k}\right) \rightarrow 0$, and $u_{k} \rightarrow v$ in $L_{\mathrm{loc}}^{p}(\Omega)$. In the latter case, $v$ is (up to a multiplicative constant) the unique positive supersolution of the equation $Q^{\prime}(u)=0$ in $\Omega$, and one has for $Q$ an inequality of Poincaré type: there exists a positive continuous function $W$ such that for every $\psi \in C_{0}^{\infty}(\Omega)$ satisfying $\int \psi v \mathrm{~d} x \neq 0$ there exists a constant $C>0$ such that $C^{-1} \int W|u|^{p} \mathrm{~d} x \leq Q(u)+C\left|\int u \psi \mathrm{d} x\right|^{p}$ for all $u \in C_{0}^{\infty}(\Omega)$. As a consequence, we prove positivity properties for the quasilinear operator $Q^{\prime}$ that are known to hold for general subcritical resp. critical second-order linear elliptic operators.
\end{abstract}

2000 Mathematics Subject Classification. Primary 35J20; Secondary 35J60, 35J70, 49R50.

Keywords. quasilinear elliptic operator, $p$-Laplacian, ground state, positive solutions, Green function, isolated singularity. 


\section{Introduction}

Positivity properties of quasilinear elliptic equations, in particular those with the $p$-Laplacian term in the principal part, have been extensively studied over the recent decades (see for example [2, 3, 4, 6, 7, 11, 12, 15, 16, 21, 25, 33, 38, and the references therein). The objective of the present paper is to study general positivity properties of such equations defined on general domains in $\mathbb{R}^{d}$. We generalize some results, obtained for $p=2$ in [32], to the case of $p \in(1, \infty)$. In particular, we extend to the case of the $p$-Laplacian the dichotomy of [32] obtained for nonnegative Schrödinger operators which states that either the associated quadratic form has a weighted spectral gap or the operator admits a unique ground state.

Some of the proofs in this paper (in particular, the uniqueness of a global positive supersolution for a critical operator) seem to be new even for the previously studied case $p=2$.

Fix $p \in(1, \infty)$, a domain $\Omega \subseteq \mathbb{R}^{d}$, and a potential $V \in L_{\text {loc }}^{\infty}(\Omega)$. We denote by $\Delta_{p}(u):=\nabla \cdot\left(|\nabla u|^{p-2} \nabla u\right)$ the $p$-Laplacian. Throughout this paper we assume that

$$
Q(u):=\int_{\Omega}\left(|\nabla u|^{p}+V|u|^{p}\right) \mathrm{d} x \geq 0
$$

for all $u \in C_{0}^{\infty}(\Omega)$.

Definition 1.1. We say that a function $v \in W_{\text {loc }}^{1, p}(\Omega)$ is a (weak) solution of the equation

$$
\frac{1}{p} Q^{\prime}(v):=-\Delta_{p}(v)+V|v|^{p-2} v=0 \quad \text { in } \Omega,
$$

if for every $\varphi \in C_{0}^{\infty}(\Omega)$

$$
\int_{\Omega}\left(|\nabla v|^{p-2} \nabla v \cdot \nabla \varphi+V|v|^{p-2} v \varphi\right) \mathrm{d} x=0 .
$$

We say that a positive function $v \in C_{\mathrm{loc}}^{1}(\Omega)$ is a positive supersolution of the equation (1.2) if for every nonnegative $\varphi \in C_{0}^{\infty}(\Omega)$

$$
\int_{\Omega}\left(|\nabla v|^{p-2} \nabla v \cdot \nabla \varphi+V|v|^{p-2} v \varphi\right) \mathrm{d} x \geq 0 .
$$

Remark 1.2. It is well-known that any weak solution of (1.2) admits Hölder continuous first derivatives, and that any nonnegative solution of (1.2) satisfies the Harnack inequality [10, 36, 37]. 
Definition 1.3. We say that the functional $Q$ has a weighted spectral gap in $\Omega$ (or $Q$ is strictly positive in $\Omega$ ) if there is a positive continuous function $W$ in $\Omega$ such that

$$
Q(u) \geq \int_{\Omega} W|u|^{p} \mathrm{~d} x \quad \forall u \in C_{0}^{\infty}(\Omega) .
$$

Definition 1.4. We say that a sequence $\left\{u_{k}\right\} \subset C_{0}^{\infty}(\Omega)$ is a null sequence, if $u_{k} \geq 0$ for all $k \in \mathbb{N}$, and there exists an open set $B \Subset \Omega$ (i.e., $\bar{B}$ is compact in $\Omega$ ) such that $\int_{B}\left|u_{k}\right|^{p} \mathrm{~d} x=1$, and

$$
\lim _{k \rightarrow \infty} Q\left(u_{k}\right)=\lim _{k \rightarrow \infty} \int_{\Omega}\left(\left|\nabla u_{k}\right|^{p}+V\left|u_{k}\right|^{p}\right) \mathrm{d} x=0 .
$$

We say that a positive function $v \in C_{\text {loc }}^{1}(\Omega)$ is a ground state of the functional $Q$ in $\Omega$ if $v$ is an $L_{\mathrm{loc}}^{p}(\Omega)$ limit of a null sequence. If $Q \geq 0$, and $Q$ admits a ground state in $\Omega$, we say that $Q$ is degenerately positive in $\Omega$.

Definition 1.5. The functional $Q$ is nonpositive in $\Omega$ if it takes negative values on $C_{0}^{\infty}(\Omega)$.

The main result of the present paper reads as follows:

Theorem 1.6. Let $\Omega \subseteq \mathbb{R}^{d}$ be a domain, $V \in L_{\mathrm{loc}}^{\infty}(\Omega)$, and $p \in(1, \infty)$. Suppose that the functional $Q$ is nonnegative. Then

(a) Q has either a weighted spectral gap or a ground state.

(b) If the functional $Q$ admits a ground state $v$, then $v$ satisfies (1.2).

(c) The functional $Q$ admits a ground state if and only if (1.2) admits a unique positive supersolution.

(d) If $Q$ has a ground state $v$, then there exists a positive continuous function $W$ in $\Omega$, such that for every $\psi \in C_{0}^{\infty}(\Omega)$ satisfying $\int \psi v \mathrm{~d} x \neq 0$ there exists a constant $C>0$ such that the following inequality holds:

$$
C^{-1} \int_{\Omega} W|u|^{p} \mathrm{~d} x \leq Q(u)+C\left|\int_{\Omega} \psi u \mathrm{~d} x\right|^{p} \quad \forall u \in C_{0}^{\infty}(\Omega) .
$$

Theorem 1.6 extends [32, Theorem 1.5] that deals with the linear case $p=2$. 
The outline of the present paper is as follows. The next section gives preliminary results concerning positive solutions of the equation $Q^{\prime}(u)=0$. In particular, it introduces the generalized Picone's identity [2, 3] which is a crucial tool in our study. Section 3 is devoted to the proof of Theorem 1.6. In Section 4, we study, using Theorem 1.6, criticality properties of the functional $Q$ along the lines of criticality theory for second-order linear elliptic operators [27, 28].

In Section 5 we prove, for $1<p \leq d$, the existence of a unique (up to a multiplicative constant) positive solution of the equation $Q^{\prime}(u)=0$ in $\Omega \backslash\left\{x_{0}\right\}$ which has a minimal growth in a neighborhood of infinity in $\Omega$. The proof of the above result relies on an unpublished lemma of L. Véron (Lemma 5.1) concerning the exact asymptotic behavior of a singular positive solution of the equation $Q^{\prime}(u)=0$ in a punctured neighborhood of $x_{0}$. We thank Professor Véron for kindly supplying the proof of this key result. The study of positive solutions which has a minimal growth in a neighborhood of infinity in $\Omega$ leads us to another characterization of strict positivity in terms of these solutions.

In Section 6, we pose a number of open problems suggested by the results of the present paper. The Appendix contains a new energy estimate for the functional $Q$ valid for $p>2$. This estimate leads to an alternative proof of Lemma 3.2 for the case $p>2$.

\section{Positive solutions and Picone identity}

Let $v>0, v \in C_{\text {loc }}^{1}(\Omega)$, and $u \geq 0, u \in C_{0}^{\infty}(\Omega)$. Denote

$$
R(u, v):=|\nabla u|^{p}-\nabla\left(\frac{u^{p}}{v^{p-1}}\right) \cdot|\nabla v|^{p-2} \nabla v,
$$

and

$$
L(u, v):=|\nabla u|^{p}+(p-1) \frac{u^{p}}{v^{p}}|\nabla v|^{p}-p \frac{u^{p-1}}{v^{p-1}} \nabla u \cdot|\nabla v|^{p-2} \nabla v .
$$

Then the following (generalized) Picone identity holds [2, 3]

$$
R(u, v)=L(u, v)
$$

Write $L(u, v)=L_{1}(u, v)+L_{2}(u, v)$, where

$$
L_{1}(u, v):=|\nabla u|^{p}+(p-1) \frac{u^{p}}{v^{p}}|\nabla v|^{p}-p \frac{u^{p-1}}{v^{p-1}}|\nabla u||\nabla v|^{p-1},
$$


and

$$
L_{2}(u, v):=p \frac{u^{p-1}}{v^{p-1}}|\nabla v|^{p-2}(|\nabla u||\nabla v|-\nabla u \cdot \nabla v) \geq 0 .
$$

From the obvious inequality $t^{p}+(p-1)-p t \geq 0$, we also have that $L_{1}(u, v) \geq$ 0 . Therefore, $L(u, v) \geq 0$ in $\Omega$.

Let $v \in C_{\text {loc }}^{1}(\Omega)$ be a positive solution (resp. supersolution) of (1.2). Using (2.3) and (1.3) (resp. (1.4)), we infer that for every $u \in C_{0}^{\infty}(\Omega), u \geq 0$,

$$
Q(u)=\int_{\Omega} L(u, v) \mathrm{d} x \geq 0, \quad\left(\operatorname{resp} . Q(u) \geq \int_{\Omega} L(u, v) \mathrm{d} x \geq 0\right) .
$$

For any smooth subdomain $\Omega^{\prime} \Subset \Omega$ consider the variational problem

$$
\lambda_{1, p}\left(\Omega^{\prime}\right):=\inf _{u \in W_{0}^{1, p}\left(\Omega^{\prime}\right)} \frac{\int_{\Omega^{\prime}}\left(|\nabla u|^{p}+V|u|^{p}\right) \mathrm{d} x}{\int_{\Omega^{\prime}}|u|^{p} \mathrm{~d} x} .
$$

It is well-known that for such a subdomain, (2.7) admits (up to a multiplicative constant) a unique minimizer $\varphi[12,17]$. Moreover, $\varphi$ is a positive solution of the quasilinear eigenvalue problem

$$
\begin{cases}Q^{\prime}(\varphi)=\lambda_{1, p}\left(\Omega^{\prime}\right)|\varphi|^{p-2} \varphi & \text { in } \Omega^{\prime} \\ \varphi=0 & \text { on } \partial \Omega^{\prime}\end{cases}
$$

$\lambda_{1, p}\left(\Omega^{\prime}\right)$ and $\varphi$ are called the principal eigenvalue and eigenfunction of the operator $Q^{\prime}$, respectively.

The following theorem was proved by J. García-Melián, and J. Sabina de Lis [17] (see also [2, 3]).

Theorem 2.1. Assume that $\Omega \subset \mathbb{R}^{d}$ is a bounded $C^{1+\alpha}$-domain, $0<\alpha<1$, and suppose that $V \in L^{\infty}(\Omega)$. Then the following assertions are equivalent

(i) $Q^{\prime}$ satisfies the maximum principle: If $u$ is a solution of the equation $Q^{\prime}(u)=f \geq 0$ in $\Omega$ with some $f \in L^{\infty}(\Omega)$, and satisfies $u \geq 0$ on $\partial \Omega$, then $u$ is nonnegative in $\Omega$.

(ii) $Q^{\prime}$ satisfies the strong maximum principle: If $u$ is a solution of the equation $Q^{\prime}(u)=f \supsetneqq 0$ in $\Omega$ with some $f \in L^{\infty}(\Omega)$, and satisfies $u \geq 0$ on $\partial \Omega$, then $u>0$ in $\Omega$.

(iii) $\lambda_{1, p}(\Omega)>0$. 
(iv) For some $0 \supsetneqq f \in L^{\infty}(\Omega)$ there exists a positive strict supersolution $v$ satisfying $Q^{\prime}(v)=f$ in $\Omega$, and $v=0$ on $\partial \Omega$.

(iv') There exists a positive strict supersolution $v$ satisfying $Q^{\prime}(v)=f \supsetneqq 0$ in $\Omega$, such that $v \in C^{1+\alpha}(\partial \Omega)$ and $f \in L^{\infty}(\Omega)$.

(v) For each nonnegative $f \in C^{\alpha}(\Omega) \cap L^{\infty}(\Omega)$ there exists a unique weak nonnegative solution of the problem $Q^{\prime}(u)=f$ in $\Omega$, and $u=0$ on $\partial \Omega$.

We shall need also the following comparison principle of J. García-Melián, and J. Sabina de Lis [17, Theorem 5].

Theorem 2.2. Let $\Omega \subset \mathbb{R}^{d}$ be a bounded domain of class $C^{1, \alpha}, 0<\alpha \leq 1$. Assume that $\lambda_{1, p}(\Omega)>0$ and let $u_{i} \in W^{1, p}(\Omega) \cap L^{\infty}(\Omega)$ satisfying $Q^{\prime}\left(u_{i}\right) \in$ $L^{\infty}(\Omega),\left.u_{i}\right|_{\partial \Omega} \in C^{1+\alpha}(\partial \Omega)$, where $i=1,2$. Suppose further that the following inequalities are satisfied

$$
\left\{\begin{aligned}
Q^{\prime}\left(u_{1}\right) & \leq Q^{\prime}\left(u_{2}\right) & & \text { in } \Omega, \\
Q^{\prime}\left(u_{2}\right) & \geq 0 & & \text { in } \Omega, \\
u_{1} & \leq u_{2} & & \text { on } \partial \Omega, \\
u_{2} & \geq 0 & & \text { on } \partial \Omega .
\end{aligned}\right.
$$

Then

$$
u_{1} \leq u_{2} \quad \text { in } \Omega \text {. }
$$

The following theorem generalizes the well-known Allegretto-Piepenbrink theorem (see [8, Theorem 2.12] and the references therein).

Theorem 2.3. Let $Q$ be a functional of the form (1.1). Then the following assertions are equivalent

(i) The functional $Q$ is nonnegative on $C_{0}^{\infty}(\Omega)$.

(ii) Equation (1.2) admits a global positive solution.

(iii) Equation (1.2) admits a global positive supersolution.

Proof. (i) $\Rightarrow$ (ii): Assume that $Q \geq 0$ on $C_{0}^{\infty}(\Omega)$. Then $Q$ is nonnegative on $C_{0}^{\infty}\left(\Omega^{\prime}\right)$ for any smooth bounded domain $\Omega^{\prime} \Subset \Omega$. Fix an exhaustion $\left\{\Omega_{N}\right\}_{N=1}^{\infty}$ of $\Omega$ (i.e., a sequence of smooth, relatively compact domains such that $x_{0} \in \Omega_{1}, \operatorname{cl}\left(\Omega_{N}\right) \subset \Omega_{N+1}$ and $\left.\cup_{N=1}^{\infty} \Omega_{N}=\Omega\right)$. By the strict monotonicity of $\lambda_{1, p}(\Omega)$ as a function of $\Omega$ [2, Theorem 2.3], it follows that $\lambda_{1, p}\left(\Omega_{N}\right)>0$ 
for all $N \geq 1$. Let $f_{N} \in C_{0}^{\infty}\left(\Omega_{N} \backslash \Omega_{N-1}\right)$ be a nonnegative nonzero function. By Theorem 2.1, there exists a unique positive solution of the problem

$$
Q^{\prime}\left(u_{N}\right)=f_{N} \text { in } \Omega_{N}, \quad u_{N}=0 \text { on } \partial \Omega_{N} .
$$

Set $v_{N}(x):=u_{N}(x) / u_{N}\left(x_{0}\right)$. By Harnack's inequality and elliptic regularity [34, 37], it follows that $\left\{v_{N}\right\}$ admits a subsequence which converges locally uniformly to a positive solution $v$ of the equation $Q^{\prime}(u)=0$ in $\Omega$.

(ii) $\Rightarrow$ (iii) is trivial.

(iii) $\Rightarrow$ (i): Suppose that (1.2) admits a global positive supersolution $v$ in $\Omega$. If $u \in C_{0}^{\infty}(\Omega)$ is a nonnegative function, then by (2.6) $Q(u) \geq 0$. Let $u \in W_{\text {loc }}^{1, p}(\Omega)$ be a nonnegative function with compact support. By taking a sequence $\left\{u_{k}\right\} \subset C_{0}^{\infty}(\Omega)$ of nonnegative functions such that $u_{k} \rightarrow u$ in $W_{\text {loc }}^{1, p}(\Omega)$, we infer that $Q(u) \geq 0$. Thus, $Q \geq 0$ on the cone of all $W_{\text {loc }}^{1, p}(\Omega)$ nonnegative functions with compact support in $\Omega$. Since $Q(u)=Q(|u|)$ on $C_{0}^{\infty}(\Omega)$, it follows that $Q$ is nonnegative on $C_{0}^{\infty}(\Omega)$.

\section{Proof of Theorem 1.6}

We shall start with the following two lemmas. For $B \subset \Omega$ let

$$
c_{B}:=\inf _{\substack{u \in C_{0}^{\infty}(\Omega) \\ \int_{B}|u|^{p} \mathrm{~d} x=1}} Q(u)=\inf _{\substack{0 \leq u \in C_{0}^{\infty}(\Omega) \\ \int_{B}|u|^{p} \mathrm{~d} x=1}} Q(u) .
$$

Lemma 3.1. If for every open set $B \Subset \Omega, c_{B}>0$, then there exists a $W \in C(\Omega), W>0$, such that

$$
Q(u) \geq \int_{\Omega} W(x)|u(x)|^{p} \mathrm{~d} x \quad \forall u \in C_{0}^{\infty}(\Omega) .
$$

In other words, $Q$ has a weighted spectral gap with the weight $W$.

Proof. Let $\left\{B_{j}\right\}_{j=1}^{\infty}$ be a locally finite covering of $\Omega$ by open balls $B_{i} \Subset \Omega$, and let $\left\{\chi_{j}\right\}$ be a locally finite partition of unity on $\Omega$ subordinated to this covering. Set $C_{j}:=\min \left\{c_{B_{j}}, 1\right\}$. Then

$$
2^{-j} Q(u) \geq 2^{-j} c_{B_{j}} \int_{B_{j}}|u|^{p} \mathrm{~d} x \geq 2^{-j} C_{j} \int_{\Omega} \chi_{j}|u|^{p} \mathrm{~d} x \quad \forall u \in C_{0}^{\infty}(\Omega) .
$$

By summation (3.3) over $j \in \mathbb{N}$ and by interchanging the order of summation and integration we obtain (3.2) with $W(x):=\sum_{j=1}^{\infty} 2^{-j} C_{j} \chi_{j}(x)$. 
Lemma 3.2. If there exists a nonempty open set $B \Subset \Omega$ such that $c_{B}=0$, then $Q$ admits a ground state.

Proof. Fix a positive (super)solution $v \in C^{1}(\Omega)$. Since $c_{B}=0$, there exists a sequence $\left\{u_{k}\right\} \subset C_{0}^{\infty}(\Omega), u_{k} \geq 0$, such that $\int_{B}\left|u_{k}\right|^{p} \mathrm{~d} x=1$ and $Q\left(u_{k}\right) \rightarrow 0$. Let $\omega \Subset \Omega$ be an open connected set containing $B$.

Step 1. Let $\omega^{\prime} \subset \omega$. By (2.6), and since $L\left(u_{k}, v\right) \geq 0$, we have that

$$
\begin{array}{r}
\int_{\omega^{\prime}}\left|\nabla u_{k}\right|^{p} \mathrm{~d} x \leq \int_{\Omega} L\left(u_{k}, v\right) \mathrm{d} x+\int_{\omega^{\prime}} C u_{k}^{p-1}\left|\nabla u_{k}\right| \mathrm{d} x \leq \\
o(1)+C \int_{\omega^{\prime}} u_{k}^{p-1}\left|\nabla u_{k}\right| \mathrm{d} x,
\end{array}
$$

with the constant $C=C(\omega)$ independent of $\omega^{\prime}$. Invoking Young's inequality, we arrive at

$$
\int_{\omega^{\prime}}\left|\nabla u_{k}\right|^{p} \mathrm{~d} x \leq o(1)+\frac{1}{2} \int_{\omega^{\prime}}\left|\nabla u_{k}\right|^{p} \mathrm{~d} x+C \int_{\omega^{\prime}} u_{k}^{p} \mathrm{~d} x .
$$

Therefore,

$$
\int_{\omega^{\prime}}\left|\nabla u_{k}\right|^{p} \mathrm{~d} x \leq C(\omega) \int_{\omega^{\prime}} u_{k}^{p} \mathrm{~d} x+o(1)
$$

Step 2. Let

$$
\omega_{0}:=\left\{x \in \omega: \exists \rho(x) \in(0, d(x, \Omega \backslash \omega)), \sup _{k} \int_{B_{\rho(x)}(x)}\left|u_{k}\right|^{p} \mathrm{~d} x<\infty\right\} .
$$

Since $\int_{B}\left|u_{k}\right|^{p} \mathrm{~d} x=1<\infty, B \subset \omega_{0}$. Moreover, $\omega_{0}$ is an open set. Indeed, let $x \in \omega_{0}, B_{\rho(x)}(x) \subset \omega_{0}$. Then for every point $y \in B_{\rho(x)}(x)$ there is a $\rho(y)>0$ such that $B_{\rho(y)}(y) \subset B_{\rho(x)}(x)$. Therefore, $\int_{B_{\rho(y)}(y)}\left|u_{k}\right|^{p} \mathrm{~d} x \leq \int_{B_{\rho(x)}(x)}\left|u_{k}\right|^{p} \mathrm{~d} x$. Consequently $y \in \omega_{0}$, and $\omega_{0}$ is open.

Let us show now that $\omega_{0}$ is a relatively closed set in $\omega$. We shall use the following version of Poincaré inequality

$$
\int_{B_{1}(0)}|u|^{p} \mathrm{~d} x \leq C \int_{B_{1}(0)}|\nabla u|^{p} \mathrm{~d} x+C_{r}\left|\int_{B_{r}(0)} u \mathrm{~d} x\right|^{p} \quad \forall r \in(0,1), \forall u \in C^{\infty}\left(\mathbb{R}^{d}\right),
$$


which follows for example from [39, Theorem 4.2.1]. It easily follows from (3.8) that for every $\epsilon>0$ and $\rho>0$ there exist $\delta_{\epsilon}>0$ and $C(\epsilon, \rho)$ such that for every $x \in \omega, \delta \in\left(0, \min \left\{\delta_{\epsilon}, d(x, \partial \omega)\right\}\right)$, and $u \in C_{0}^{\infty}(\Omega)$,

$$
\int_{B_{\delta}(x)}|u|^{p} \mathrm{~d} x \leq \epsilon \int_{B_{\delta}(x)}|\nabla u|^{p} \mathrm{~d} x+C(\epsilon, \rho) \int_{B_{\rho}(x)}|u|^{p} \mathrm{~d} x .
$$

Let $x_{j} \in \omega_{0}, x_{j} \rightarrow x_{0} \in \omega$. Let $\epsilon<(C(\omega))^{-1} / 2$, where $C(\omega)$ is the constant in (3.6). Let $\delta_{\epsilon}>0$ be as in (3.9) and fix $\delta \in\left(0, \min \left\{\delta_{\epsilon}, d(x, \partial \omega)\right\}\right)$. Finally, choose $j$ such that $\left|x_{0}-x_{j}\right| \leq \frac{\delta}{2}$. Then, with $\rho=\rho\left(x_{j}\right)$, (3.6) and (3.9) imply

$$
\frac{1}{2} C(\omega)^{-1} \int_{B_{\delta}\left(x_{j}\right)}\left|\nabla u_{k}\right|^{p} \mathrm{~d} x \leq C\left(\epsilon, \rho\left(x_{j}\right)\right) \int_{B_{\rho\left(x_{j}\right)}\left(x_{j}\right)}\left|u_{k}\right|^{p} \mathrm{~d} x+o(1) .
$$

The right hand side of (3.10) is bounded in $k$ by the definition of $\omega_{0}$. Thus $\int_{B_{\delta}\left(x_{j}\right)}\left|\nabla u_{k}\right|^{p} \mathrm{~d} x$ is bounded, and by (3.9), $\int_{B_{\delta}\left(x_{j}\right)}\left|u_{k}\right|^{p} \mathrm{~d} x$ is also bounded. Since, by the choice of $j, B_{\delta / 2}\left(x_{0}\right) \subset B_{\delta}\left(x_{j}\right)$, it follows that $\int_{B_{\delta / 2}\left(x_{0}\right)}\left(\left|\nabla u_{k}\right|^{p}+\right.$ $\left.\left|u_{k}\right|^{p}\right) \mathrm{d} x$ is bounded and consequently, $x_{0} \in \omega_{0}$, and $\omega_{0}$ is also relatively closed.

Since $\omega$ is connected and $\omega_{0} \neq \emptyset$, it follows that $\omega_{0}=\omega$ and $\left\{u_{k}\right\}$ is bounded in $L_{\mathrm{loc}}^{p}(\Omega)$. Invoking again (3.6) it follows that $\left\{u_{k}\right\}$ is bounded in $W_{\text {loc }}^{1, p}(\Omega)$.

Step 3. Consider now a weakly convergent renamed subsequence $u_{k} \rightarrow u$ in $W_{\text {loc }}^{1, p}(\Omega)$. Let $\omega \Subset \Omega$ be a smooth domain, and set $Q^{\omega}(u):=\int_{\omega} L(u, v) \mathrm{d} x$. We claim that the functional $Q^{\omega}(u)$ is weakly lower semicontinuous in $W^{1, p}(\omega)$. Indeed, the functionals $\int_{\omega}|\nabla u|^{p} \mathrm{~d} x$ and $\int_{\omega}(p-1) \frac{|u|^{p}}{v^{p}}|\nabla v|^{p} \mathrm{~d} x$ are weakly lower semicontinuous in $W^{1, p}(\omega)$ since their Lagrangians $\left(\mathcal{L}(q, z, x)=|q|^{p}\right.$ and $\mathcal{L}(q, z, x)=(p-1) \frac{|z|^{p}}{v(x)^{p}}|\nabla v(x)|^{p}$, resp.) are convex functions of $q$. So, it suffices to show that the functional

$$
J^{\omega}(u):=\int_{\omega} \frac{u^{p-1}}{v^{p-1}} \nabla u \cdot|\nabla v|^{p-2} \nabla v \mathrm{~d} x
$$

is weakly continuous on any sequence $\left\{u_{k}\right\}$ satisfying $u_{k} \rightarrow u$ in $W^{1, p}(\omega)$. Indeed,

$$
\begin{array}{r}
J^{\omega}\left(u_{k}\right)-J^{\omega}(u)=\int_{\omega}|\nabla v|^{p-2} v^{1-p} \nabla v \cdot \nabla u_{k}\left(u_{k}^{p-1}-u^{p-1}\right) \mathrm{d} x+ \\
\int_{\omega} \nabla\left(u_{k}-u\right) \cdot \nabla v|\nabla v|^{p-2} v^{1-p} u^{p-1} \mathrm{~d} x .
\end{array}
$$


Consider the first term of the right hand side of (3.12). Since $u_{k} \rightarrow u$ in $W^{1, p}(\omega)$, it follows by the compactness of the local Sobolev imbeddings that (up to a subsequence) $u_{k} \rightarrow u$ in $L^{p}(\omega)$. Then, for a renamed subsequence, there exists a $U \in L^{p}(\omega)$, such that $0 \leq u_{k} \leq U$ and $u_{k} \rightarrow u$ a.e. in $\omega$. Therefore, $u_{k}^{p-1} \leq U^{p-1} \in L^{p^{\prime}}(\omega)$, where $p^{\prime}:=p /(p-1)$ is the conjugate exponent of $p$. Consequently,

$$
\left|u_{k}^{p-1}-u^{p-1}\right|^{p^{\prime}} \leq C\left(U^{p}+u^{p}\right) \in L^{1}(\omega) .
$$

Hence by Hölder's inequality and Lebesgue's dominated convergence theorem,

$$
\left.\left|\int_{\omega}\right| v\right|^{p-2} v^{1-p} \nabla v \cdot \nabla u_{k}\left(u_{k}^{p-1}-u^{p-1}\right) \mathrm{d} x \mid \leq C\left\|\nabla u_{k}\right\|\left\|_{p}\right\| u_{k}^{p-1}-u^{p-1} \|_{p^{\prime}} \rightarrow 0 .
$$

Consider the functional

$$
\Phi(w):=\int_{\omega} \nabla w \cdot \nabla v|\nabla v|^{p-2} v^{1-p} u^{p-1} \mathrm{~d} x .
$$

Note $|\nabla v|^{p-1} v^{1-p} u^{p-1} \in L^{p^{\prime}}(\omega)$. Therefore, Hölder's inequality implies that $\Phi$ is a continuous functional on $W^{1, p}(\omega)$. Hence, by the definition of weak convergence, the second term of the right hand side of (3.12) converges to zero.

We conclude that $0 \leq Q^{\omega}(u) \leq \liminf Q^{\omega}\left(u_{k}\right)=0$. Moreover, $\int_{B} u^{p} \mathrm{~d} x=$ 1. Now we repeat the argument of [2]. Since $Q^{\omega}(u)=0$ for every subdomain $\omega \Subset \Omega$ containing $B$, it follows that $L_{1}(u, v)=0$ and $L_{2}(u, v)=0$. Recall that $f(t)=t^{p}+p-1-p t$ is a nonnegative function on $\mathbb{R}_{+}$which attains its zero minimum only at $t=1$. Therefore, $L_{1}(u, v)=0$ implies that $u^{-1}|\nabla u|=$ $v^{-1}|\nabla v|$. On the other hand, $L_{2}(u, v)=0$ implies that $\nabla u$ is parallel to $\nabla v$. Hence, $u=c v$, where $c>0$. The value of $c$ is determined by the condition $\int_{B}(c v)^{p} \mathrm{~d} x=1$. Therefore $c v$ is the limit of every weakly convergent subsequence of $\left\{u_{k}\right\}$. It follows that the original sequence $u_{k} \rightarrow c v$ in $L_{\text {loc }}^{p}(\Omega)$.

Remark 3.3. The argument in Step 3 of the proof of Lemma 3.2 shows that the functional $Q$ has some weakly lower semicontinuity properties, as Proposition 3.5 below demonstrates.

First, we need to extend the definition of the functional $Q$ from $C_{0}^{\infty}(\Omega)$ to a larger set in $W_{\text {loc }}^{1, p}(\Omega)$. 
Definition 3.4. Let $v$ be a positive solution of the equation $Q^{\prime}(u)=0$ in $\Omega$. We define a functional $Q_{v}: W_{\text {loc }}^{1, p}(\Omega) \rightarrow[0,+\infty]$ by

$$
Q_{v}(u):= \begin{cases}\int_{\Omega} L(|u|, v) \mathrm{d} x & \text { if this integral is finite } \\ \infty & \text { otherwise. }\end{cases}
$$

Also, for $u \in W_{\text {loc }}^{1, p}(\Omega)$ and any $\omega \Subset \Omega$ define $Q_{v}^{\omega}(u):=\int_{\omega} L(|u|, v) \mathrm{d} x$.

We have

Proposition 3.5. Let

$$
\begin{array}{r}
D:=\left\{u \in W_{\mathrm{loc}}^{1, p}(\Omega) \mid Q_{v}(u)<\infty, \text { and } \exists\left\{u_{k}\right\} \subset \cup_{\omega \Subset \Omega} W_{0}^{1, p}(\omega)\right. \text { s.t. } \\
\left.u_{k} \rightarrow u \text { in } W_{\mathrm{loc}}^{1, p}(\Omega), \text { and } Q_{v}\left(u_{k}\right) \rightarrow Q_{v}(u)\right\} .
\end{array}
$$

Then the functional $Q$ on $C_{0}^{\infty}(\Omega)$ admits an extension to the set $D$ given by $Q(u):=Q_{v}(u)$. This extension is independent of the positive solution $v$. Moreover, the functional $Q$ is continuous on $W_{0}^{1, p}(\omega) \subset D$ for every $\omega \Subset \Omega$, and is weakly lower semicontinuous in the following sense:

$$
u_{k}, u \in D, u_{k} \rightarrow u \text { in } W_{\text {loc }}^{1, p}(\Omega), \sup _{k \in \mathbb{N}} Q\left(u_{k}\right)<\infty \Rightarrow \liminf _{k \rightarrow \infty} Q\left(u_{k}\right) \geq Q(u) .
$$

Note that the functional $Q^{1 / p}$ is generally not a norm (see the discussion prior to Problem 6.1). If $Q^{1 / p}$ is a norm and $Q$ is strictly positive, then the set $D$ is a closure of $C_{0}^{\infty}(\Omega)$ in that norm and so it is a Banach space. In this case, the condition in (3.16) is equivalent to weak convergence in $D$, and $Q$ is weakly lower semicontinuous as a monotone increasing function of the norm.

Proof. The functionals $Q$ and $Q_{v}$ obviously admit continuous extensions to $W_{0}^{1, p}(\omega)$ for every $\omega \Subset \Omega$. Since $Q$ and $Q_{v}$ are even and coincide on nonnegative $C_{0}^{\infty}(\Omega)$-functions, their respective extensions to $W_{0}^{1, p}(\omega)$ are equal. Consequently, the set $D$ is independent of $v$. Moreover, the functional $Q_{v}$ evaluated on $D$ is independent of $v$ and thus defines the extension of $Q$.

Let $u_{k} \rightarrow u$ in $W_{\text {loc }}^{1, p}(\Omega), u_{k}, u \in D$, and $Q_{v}\left(u_{k}\right) \leq C$ for some $C>0$. Assume first that $u_{k} \geq 0$. Then $Q_{v}\left(u_{k}\right) \geq Q_{v}^{\omega}\left(u_{k}\right)$ for every open $\omega \Subset \Omega$. Step 3 of Lemma 3.2 implies that $\liminf Q_{v}\left(u_{k}\right) \geq \liminf Q_{v}^{\omega}\left(u_{k}\right) \geq Q_{v}^{\omega}(u)$. Since $\omega$ is arbitrary, we have $\lim \inf Q_{v}\left(u_{k}\right) \geq Q_{v}(u)=Q(u)$. 
Let now remove the restriction $u_{k} \geq 0$. Let $\omega \Subset \Omega$ be a smooth domain. Due to the compactness of the imbedding of $W^{1, p}(\omega)$ into $L^{p}(\omega), u_{k} \rightarrow u$ a.e. on a renamed subsequence, and $\left|u_{k}\right| \leq U$ with some $U \in L_{\text {loc }}^{p}(\Omega)$. Consequently $\left|u_{k}\right| \rightarrow|u|$ in $L_{\text {loc }}^{p}(\Omega)$. Since $\left|u_{k}\right|$ is bounded with respect to the seminorms of $W_{\text {loc }}^{1, p}(\Omega),\left|u_{k}\right| \rightarrow|u|$ in $W_{\text {loc }}^{1, p}(\Omega)$. Since the functional $Q_{v}$ is even, by the previous argument it follows that

$$
\liminf _{k \rightarrow \infty} Q_{v}\left(u_{k}\right)=\liminf _{k \rightarrow \infty} Q_{v}\left(\left|u_{k}\right|\right) \geq Q_{v}(|u|)=Q_{v}(u)=Q(u),
$$

and the proposition is proved.

Proof of Theorem 1.6. Part (a) follows from Lemma 3.1] and Lemma 3.2. To prove (b) and (c), observe that from Lemma 3.2 it follows that for each positive supersolution $v$ of (1.2), any null sequence $\left\{u_{k}\right\}$ converges to a constant multiple of $v$. This implies that all positive supersolutions of (1.2) are scalar multiples of the same function. On the other hand, if $Q$ admits a weighted spectral gap with a weight $W$, then by Theorem 2.3 , the equation $\frac{1}{p} Q^{\prime}(u)-W|u|^{p-2} u=0$ admits a positive solution $v$ in $\Omega$. So, $v$ is a strictly positive supersolution of the equation $Q^{\prime}(u)=0$ in $\Omega$. In addition, by Theorem 2.3, the equation $Q^{\prime}(u)=0$ admits a positive solution $w$ in $\Omega$. Clearly $v \neq w$, and the equation $Q^{\prime}(u)=0$ admits two linearly independent positive supersolutions in $\Omega$.

It remains to prove (d). First we claim that for every open set $B \Subset \Omega$ there is a strictly positive continuous function $W$ such that

$$
\int_{\Omega} W|u|^{p} \mathrm{~d} x \leq Q(u)+\int_{B}|u|^{p} \mathrm{~d} x \quad \forall u \in C_{0}^{\infty}(\Omega) .
$$

Indeed, denote the functional in the right hand side of (3.17) by $\tilde{Q}$. Clearly, $\tilde{Q} \geq 0$ on $C_{0}^{\infty}(\Omega)$. Suppose that $\tilde{Q}$ admits a null sequence $\left\{u_{k}\right\}$, then $\left\{u_{k}\right\}$ is a null sequence of $Q$, which implies $\int_{B}\left|u_{k}\right|^{p} \mathrm{~d} x \rightarrow \int_{B}|v|^{p} \mathrm{~d} x$, where $v$ is a ground state for $Q$. Consequently, $\lim \inf \tilde{Q}\left(u_{k}\right)>0$, which contradicts the definition of $\left\{u_{k}\right\}$. Therefore, part (a) of the present theorem implies that $\tilde{Q}$ admits a weighted spectral gap.

Therefore, in order to prove (1.7), it suffices to show that for some open $B \Subset \Omega$,

$$
\int_{B}|u|^{p} \mathrm{~d} x \leq C\left(Q(u)+\left|\int_{\Omega} u \psi \mathrm{d} x\right|^{p}\right) \quad \forall u \in C_{0}^{\infty}(\Omega) .
$$


Suppose that (3.18) fails. Then there is a sequence $\left\{u_{k}\right\} \subset C_{0}^{\infty}(\Omega)$ such that $\int_{B}\left|u_{k}\right|^{p} \mathrm{~d} x=1, Q\left(u_{k}\right) \rightarrow 0$ and $\int_{\Omega} u_{k} \psi \mathrm{d} x \rightarrow 0$. Since $\left\{u_{k}\right\}$ is a null sequence it converges in $L_{\mathrm{loc}}^{p}(\Omega)$ to $v$, where $v>0$ is the ground state of $Q$. Then $\int_{\Omega} u_{k} \psi \mathrm{d} x \rightarrow \int_{\Omega} v \psi \mathrm{d} x \neq 0$, and we arrive at a contradiction.

For $u \in C_{0}^{\infty}(\Omega)$, we define

$$
\tilde{Q}(u):= \begin{cases}Q(u) & \text { if } \mathrm{Q} \text { has a weighted spectral gap, } \\ Q(u)+C\left|\int_{\Omega} \psi u \mathrm{~d} x\right|^{p} & \text { if } \mathrm{Q} \text { has a ground state, }\end{cases}
$$

where $C$ is the constant in (1.7).

Proposition 3.6. For any $C>0$, the set $S:=\left\{u \in C_{0}^{\infty}(\Omega) \mid u \geq 0, \tilde{Q}(u) \leq\right.$ $C\}$ is bounded in $W_{\mathrm{loc}}^{1, p}(\Omega)$ and therefore, it is relatively compact in $L_{\mathrm{loc}}^{p}(\Omega)$.

Proof. Let $u \in S$ and let $v$ be a positive solution of (1.2). Let $\omega \Subset \Omega$ be an open set. Then by (2.6), we have that

$$
\begin{array}{r}
\int_{\omega}\left(|\nabla u|^{p}+|u|^{p}\right) \mathrm{d} x \leq \int_{\omega}\left(L(u, v)+C|u|^{p-1}|\nabla u|+|u|^{p}\right) \mathrm{d} x \leq \\
C+C \int_{\omega}|u|^{p-1}|\nabla u| \mathrm{d} x+\int_{\omega}|u|^{p} \mathrm{~d} x .
\end{array}
$$

In light of Young's inequality we obtain

$$
\int_{\omega}\left(|\nabla u|^{p}+|u|^{p}\right) \mathrm{d} x \leq C+\frac{1}{2} \int_{\omega}|\nabla u|^{p} \mathrm{~d} x+C \int_{\omega}|u|^{p} \mathrm{~d} x .
$$

Consequently,

$$
\int_{\omega}\left(|\nabla u|^{p}+|u|^{p}\right) \mathrm{d} x \leq C+C \int_{\omega}|u|^{p} \mathrm{~d} x .
$$

By (1.5) or (1.7) and the definition of $S$, the right hand side of (3.19) is uniformly bounded in $S$, and therefore $S$ is a bounded set in $W_{\text {loc }}^{1, p}(\Omega)$.

\section{Criticality theory}

In this section we prove several positivity properties of the functional $Q$ along the lines of criticality theory for second-order linear elliptic operators [27, 28]. 
Recall that $Q$ is said to be strictly positive in $\Omega$ if $Q$ has a weighted spectral gap on $C_{0}^{\infty}(\Omega)$. The functional $Q$ is degenerately positive in $\Omega$ if $Q \geq 0$ on $C_{0}^{\infty}(\Omega)$ and $Q$ admits a ground state in $\Omega$. The functional $Q$ is nonpositive in $\Omega$ if it takes negative values on $C_{0}^{\infty}(\Omega)$. For $V \in L_{\text {loc }}^{\infty}(\Omega)$, we denote in the present section

$$
Q_{V}(u):=\int_{\Omega}\left(|\nabla u|^{p}+V|u|^{p}\right) \mathrm{d} x
$$

to emphasize the dependence of $Q$ on the potential $V$.

Proposition 4.1. Let $V_{i} \in L_{\mathrm{loc}}^{\infty}(\Omega)$ and suppose that $V_{2} \supsetneqq V_{1}$. If $Q_{V_{1}} \geq 0$ on $C_{0}^{\infty}(\Omega)$, then $Q_{V_{2}}$ is strictly positive in $\Omega$, and if $Q_{V_{2}}$ is degenerately positive in $\Omega$, then $Q_{V_{1}}$ is nonpositive in $\Omega$.

Proof. Obviously,

$$
Q_{V_{2}}(u)=Q_{V_{1}}(u)+\int\left(V_{2}-V_{1}\right)|u|^{p} \mathrm{~d} x \geq 0 \quad \forall u \in C_{0}^{\infty}(\Omega) .
$$

Suppose that $Q_{V_{2}}$ has a null sequence $\left\{u_{k}\right\}$ with a ground state $v$, such that $u_{k} \rightarrow v$ in $L_{\text {loc }}^{p}(\Omega)$. Evaluating the limit of $Q_{V_{2}}\left(u_{k}\right)$, we have by Fatou's lemma,

$$
0=\lim _{k \rightarrow \infty} Q_{V_{2}}\left(u_{k}\right) \geq \liminf _{k \rightarrow \infty} \int_{\Omega}\left(V_{2}-V_{1}\right)\left|u_{k}\right|^{p} \mathrm{~d} x \geq \int_{\Omega}\left(V_{2}-V_{1}\right)|v|^{p} \mathrm{~d} x>0,
$$

and we arrive at a contradiction.

Proposition 4.2. Let $\Omega_{1} \subset \Omega_{2}$ be domains in $\mathbb{R}^{d}$ such that $\Omega_{2} \backslash \overline{\Omega_{1}} \neq \emptyset$. Let $Q_{V}$ be defined on $C_{0}^{\infty}\left(\Omega_{2}\right)$.

1. If $Q_{V} \geq 0$ on $C_{0}^{\infty}\left(\Omega_{2}\right)$, then $Q_{V}$ is strictly positive in $\Omega_{1}$.

2. If $Q_{V}$ is degenerately positive in $\Omega_{1}$, then $Q_{V}$ is nonpositive in $\Omega_{2}$.

Proof. 1. If $Q_{V}$ is strictly positive in $\Omega_{2}$, then the first assertion is trivial. Suppose that $Q_{V}$ is degenerately positive in $\Omega_{2}$, and let $v$ be the ground state of $Q_{V}$ in $\Omega_{2}$. Take $\psi \in C_{0}^{\infty}\left(\Omega_{2} \backslash \overline{\Omega_{1}}\right)$ such that $\int_{\Omega_{2}} v \psi \mathrm{d} x \neq 0$.

Due to (1.7) restricted to $u \in C_{0}^{\infty}\left(\Omega_{1}\right)$, we conclude that $Q_{V}$ is strictly positive in $\Omega_{1}$.

2. Assume that $Q_{V} \geq 0$ on $C_{0}^{\infty}\left(\Omega_{2}\right)$. Then by the first part, $Q_{V}$ is strictly positive in $\Omega_{1}$, which is a contradiction. 
Proposition 4.3. Let $V_{0}, V_{1} \in L_{\mathrm{loc}}^{\infty}(\Omega), V_{0} \neq V_{1}$. For $t \in \mathbb{R}$ we denote

$$
Q_{t}(u):=t Q_{V_{1}}(u)+(1-t) Q_{V_{0}}(u),
$$

and suppose that $Q_{V_{i}} \geq 0$ on $C_{0}^{\infty}(\Omega)$ for $i=0,1$.

Then the functional $Q_{t} \geq 0$ on $C_{0}^{\infty}(\Omega)$ for all $t \in[0,1]$. Moreover, if $V_{0} \neq V_{1}$, then $Q_{t}$ is strictly positive in $\Omega$ for all $t \in(0,1)$.

Proof. The first assertion is immediate. To prove the second assertion, assume first that at least one of $Q_{V_{0}}, Q_{V_{1}}$, say, $Q_{V_{0}}$, is strictly positive with a weight $W_{0}$. Then for $0 \leq \tau<1$, the functional $Q_{\tau}$ is strictly positive with the weight $(1-\tau) W_{0}$.

Assume now that both $Q_{V_{0}}$ and $Q_{V_{1}}$ are degenerately positive with ground states $v_{0}, v_{1}$, respectively, and assume that for some $\tau \in(0,1), Q_{\tau}$ has a null sequence $\left\{u_{k}\right\}$ and a ground state $v_{\tau}$.

Note that $v_{\tau}$ is not a multiple of $v_{0}$ or of $v_{1}$ since $V_{0} \neq V_{1}$. Then there exist $\psi_{i} \in C_{0}^{\infty}(\Omega), i=0,1$, such that

$$
\int_{\Omega} \psi_{i} v_{i} \mathrm{~d} x \neq 0, \quad \text { and } \int_{\Omega} \psi_{i} v_{\tau} \mathrm{d} x=0 \quad i=0,1 .
$$

By (1.7), for $i=0,1$ there exist a continuous function $W_{i}>0$ in $\Omega$, and a constant $C_{i}>0$, such that

$$
\int_{\Omega} W_{i}|u|^{p} \mathrm{~d} x \leq Q_{i}(u)+C_{i}\left|\int_{\Omega} u \psi_{i} \mathrm{~d} x\right|^{p} \quad \forall u \in C_{0}^{\infty}(\Omega) .
$$

Let $W_{\tau}:=\tau W_{1}+(1-\tau) W_{0}$. Then

$$
\int_{\Omega} W_{\tau}|u|^{p} \mathrm{~d} x \leq Q_{\tau}(u)+C_{1} \tau\left|\int_{\Omega} u \psi_{1} \mathrm{~d} x\right|^{p}+C_{0}(1-\tau)\left|\int_{\Omega} u \psi_{0} \mathrm{~d} x\right|^{p} \quad \forall u \in C_{0}^{\infty}(\Omega) .
$$

Substituting $u=u_{k}$ and passing to the limit, taking into account that $u_{k} \rightarrow$ $v_{\tau}$ in $L_{\mathrm{loc}}^{p}(\Omega)$ as well as (4.4) and Fatou's lemma, we have

$$
0<\int_{\Omega} W_{\tau}\left|v_{\tau}\right|^{p} \mathrm{~d} x \leq C_{1} \tau\left|\int_{\Omega} v_{\tau} \psi_{1} \mathrm{~d} x\right|^{p}+C_{0}(1-\tau)\left|\int_{\Omega} v_{\tau} \psi_{0} \mathrm{~d} x\right|^{p}=0,
$$

and we arrive at a contradiction. Therefore, $Q_{\tau}$ does not admit a ground state, and by Theorem 1.6, the functional $Q_{\tau}$ is strictly positive in $\Omega$. 
Proposition 4.4. Let $Q_{V}$ be a strictly positive functional in $\Omega$. Consider $V_{0} \in L^{\infty}(\Omega)$ such that $V_{0} \ngtr 0$ and $\operatorname{supp} V_{0} \Subset \Omega$. Then there exist $\tau_{+}>0$ and $-\infty \leq \tau_{-}<0$ such that $Q_{V+t V_{0}}$ is strictly positive in $\Omega$ for $t \in\left(\tau_{-}, \tau_{+}\right)$, and $Q_{V+\tau_{+} V_{0}}$ is degenerately positive in $\Omega$.

Proof. If $Q_{V}$ has a weighted spectral gap in $\Omega$ with a continuous weight $W$, then $Q_{V+t V_{0}}$ satisfies the inequality

$$
\int_{\Omega}\left(W+t V_{0}\right)|u|^{p} \mathrm{~d} x \leq Q_{V+t V_{0}}(u) \quad \text { on } C_{0}^{\infty}(\Omega) .
$$

The weight $W+t V_{0}$ is strictly positive in $\Omega$ for $|t|$ small, since $W$ is a strictly positive continuous function and $V_{0}$ is a bounded function with compact support. By Proposition 4.3, the set of $t \in \mathbb{R}$, for which $Q_{V+t V_{0}}$ is strictly positive, is an interval. Moreover, this interval does not extend to $+\infty$. Indeed, take $u_{0} \in C_{0}^{\infty}$ such that $\int_{\Omega} V_{0}\left|u_{0}\right|^{p} \mathrm{~d} x<0$ to see that $Q_{V+t V_{0}}\left(u_{0}\right)<0$ for $t$ sufficiently large. Note that by the above argument, this interval extends to $-\infty$ if and only if $V_{0} \leq 0$.

Let $\tau_{+}$be the right endpoint of this interval. Obviously $Q_{V+\tau_{+} V_{0}}$ is nonnegative on $C_{0}^{\infty}(\Omega)$. If, on the other hand, the functional $Q_{V+\tau_{+} V_{0}}$ is strictly positive in $\Omega$, then by the preceding argument, there exists $\delta>0$ such that the functional $Q_{V+\left(\tau_{+}+\delta\right) V_{0}}$ is strictly positive in $\Omega$, which contradicts the definition of $\tau_{+}$.

Proposition 4.5. Let $Q_{V}$ be a degenerately positive functional in $\Omega$, and let $v$ be the corresponding ground state. Consider $V_{0} \in L^{\infty}(\Omega)$ such that $\operatorname{supp} V_{0} \Subset \Omega$. Then there exists $0<\tau_{+} \leq \infty$ such that $Q_{V+t V_{0}}$ is strictly positive in $\Omega$ for $t \in\left(0, \tau_{+}\right)$if and only if

$$
\int_{\Omega} V_{0}|v|^{p} \mathrm{~d} x>0
$$

Proof. Suppose that there exists $t>0$ such that $Q_{V+t V_{0}}$ is strictly positive in $\Omega$. Then there exists $W \in C(\Omega), W>0$, such that

$$
Q_{V}(u)+t \int_{\Omega} V_{0}|u|^{p} \mathrm{~d} x \geq \int_{\Omega} W|u|^{p} \mathrm{~d} x \quad \forall u \in C_{0}^{\infty}(\Omega) .
$$

Let $\left\{u_{k}\right\}$ be a null sequence for the functional $Q_{V}$, and let $v>0$ be the ground state of $Q_{V}$ which is the $L_{\mathrm{loc}}^{p}(\Omega)$ limit of $\left\{u_{k}\right\}$. By (4.10) and Fatou's 
lemma we have

$$
\begin{array}{r}
t \int_{\Omega} V_{0}|v|^{p} \mathrm{~d} x=\lim _{k \rightarrow \infty} Q_{V}\left(u_{k}\right)+t \lim _{k \rightarrow \infty} \int_{\Omega} V_{0}\left|u_{k}\right|^{p} \mathrm{~d} x \geq \\
\liminf _{k \rightarrow \infty} \int_{\Omega} W\left|u_{k}\right|^{p} \mathrm{~d} x \geq \int_{\Omega} W|v|^{p} \mathrm{~d} x>0 .
\end{array}
$$

Thus, (4.9) is satisfied.

Suppose that (4.9) holds true, but for any $t>0$ the functional $Q_{V+t V_{0}}$ is nonpositive in $\Omega$. Therefore, for any $t>0$ there exists $u_{t} \in C_{0}^{\infty}(\Omega)$ such that

$$
Q_{V}\left(u_{t}\right)+t \int_{\Omega} V_{0}\left|u_{t}\right|^{p} \mathrm{~d} x<0
$$

Clearly, we may assume that $u_{t} \geq 0$. Since $Q_{V}\left(u_{t}\right) \geq 0$ it follows that

$$
\int_{\Omega} V_{0}\left|u_{t}\right|^{p} \mathrm{~d} x<0
$$

In particular, $\operatorname{supp}\left(u_{t}\right) \cap \operatorname{supp}\left(V_{0}\right) \neq \emptyset$. Therefore, we may assume that

$$
\int_{\operatorname{supp}\left(V_{0}\right)}\left|u_{t}\right|^{p} \mathrm{~d} x=1 \text {. }
$$

It follows that

$$
\lim _{t \rightarrow 0} t \int_{\Omega} V_{0}\left|u_{t}\right|^{p} \mathrm{~d} x=0,
$$

and by the nonnegativity of $Q_{V}$ and (4.11),

$$
0 \leq \liminf _{t \rightarrow 0} Q_{V}\left(u_{t}\right) \leq \limsup _{t \rightarrow 0} Q_{V}\left(u_{t}\right) \leq 0 .
$$

It follows that $\left\{u_{t}\right\}$ is a null sequence, and therefore, $u_{t} \rightarrow v$ in $L_{\text {loc }}^{p}(\Omega)$ as $t \rightarrow 0$, where $v$ is the corresponding ground state of $Q_{V}$. Using a standard argument similar to (3.13) we have (for a subsequence)

$$
\lim _{t \rightarrow 0} \int_{\Omega} V_{0}\left|u_{t}\right|^{p} \mathrm{~d} x=\int_{\Omega} V_{0}|v|^{p} \mathrm{~d} x .
$$

Combining (4.9), and (4.12) and (4.15), we obtain

$$
0<\int_{\Omega} V_{0}|v|^{p} \mathrm{~d} x=\lim _{t \rightarrow 0} \int_{\Omega} V_{0}\left|u_{t}\right|^{p} \mathrm{~d} x \leq 0,
$$

which is a contradiction.

Remark 4.6. An alternative proof of Proposition 4.5 can be derived from the (nonsymmetric) technique in [30]. 


\section{Minimal growth}

In this section we study the existence of positive solutions of the equation $Q^{\prime}(u)=0$ of minimal growth in a neighborhood of infinity in $\Omega$, and obtain a new characterization of strict positivity in terms of these solutions.

Throughout this section we assume that $1<p \leq d$. Therefore, for any $x_{0} \in \Omega$, any positive solution $v$ of the equation $Q^{\prime}(u)=0$ in a punctured neighborhood of $x_{0}$ has either a removable singularity at $x_{0}$, or

$$
v(x) \asymp\left\{\begin{array}{ll}
\left|x-x_{0}\right|^{\alpha(d, p)} & p<d, \\
-\log \left|x-x_{0}\right| & p=d,
\end{array} \quad \text { as } x \rightarrow x_{0},\right.
$$

where $\alpha(d, p):=(p-d) /(p-1)$ [34, 35, 38]. Here $f \asymp g$ means that $c \leq f / g \leq$ $C$, where $c$ and $C$ are positive constants. In particular, in the nonremovable case,

$$
\lim _{x \rightarrow x_{0}} v(x)=\infty
$$

Lemma 5.1 (L. Véron, private communication). Assume that $1<p \leq d$, and let $x_{0} \in \mathbb{R}^{d}$ be fixed. Suppose that $v$ is a positive solution of the equation $Q^{\prime}(u)=0$ in a punctured neighborhood of $x_{0}$ which has a nonremovable singularity at $x_{0}$. Then

$$
v(x) \sim\left\{\begin{array}{ll}
\left|x-x_{0}\right|^{\alpha(d, p)} & p<d, \\
-\log \left|x-x_{0}\right| & p=d,
\end{array} \quad \text { as } x \rightarrow x_{0},\right.
$$

where $f \sim g$ means that

$$
\lim _{x \rightarrow x_{0}} \frac{f(x)}{g(x)}=C
$$

for some positive constant $C$.

Remark 5.2. The asymptotics (5.3) has been proved for $p=2$ in [18], for $1<p \leq d$ and $V=0$ in [23, Theorem 2.1], and also in some other cases in [19]. The proof below uses a technique involving a scaling argument together with a comparison principle that has been used for example in [5].

Proof. Assume that $1<p<d$, the proof for $p=d$ needs some minor modifications, and is left to the reader. Without loss of generality, we assume also that $x_{0}=0$. 
Since $V \in L_{\text {loc }}^{\infty}(\Omega)$, the solution $v$ satisfies $v(x) \asymp|x|^{\alpha(d, p)}$. Let

$$
c:=\limsup _{x \rightarrow 0} \frac{v(x)}{|x|^{\alpha(d, p)}}=\lim _{n \rightarrow \infty} \frac{v\left(x_{n}\right)}{\left|x_{n}\right|^{\alpha(d, p)}},
$$

and set $\mu(x):=c|x|^{\alpha(d, p)}$. Define

$$
v_{n}(x):=\left|x_{n}\right|^{-\alpha(p, d)} v\left(\left|x_{n}\right| x\right),
$$

where $x_{n} \rightarrow 0$ is defined by (5.4).

Note that in an arbitrarily large punctured ball

$$
C^{-1} \mu(x) \leq v_{n}(x) \leq C \mu(x)
$$

for all $n$ large enough, and in such a ball $v_{n}$ is a positive solution of the quasilinear elliptic equation

$$
-\Delta_{p} v_{n}(x)+\left|x_{n}\right|^{p} V\left(x /\left|x_{n}\right|\right) v_{n}^{p-1}(x)=0 .
$$

Since $\left\{v_{n}\right\}$ is locally bounded and bounded away from zero in any punctured ball, a standard elliptic argument implies that there is a subsequence of $\left\{v_{n}\right\}$ that converges to a positive singular solution $U$ of the limiting equation $-\Delta_{p} U=0$ in the punctured space. Since $U \asymp \mu$ in the punctured space, it follows that $U$ tends to zero at infinity. On the other hand, [23, Theorem 2.1], implies that $U(x) \sim \mu(x)$ as $x \rightarrow 0$. Hence we can apply the comparison principle (Theorem 2.2), and compare the functions $U$ and $\mu$ on arbitrarily large balls, to obtain that $U=\mu$. This implies that

$$
\lim _{n \rightarrow \infty}\|v(x) / \mu(x)-1\|_{L^{\infty}\left(|x|=\left|x_{n}\right|\right)}=0 .
$$

In other words, $v$ is almost equal to $\mu$ on a sequence of concentric spheres converging to 0 .

In order to prove that $v$ is almost equal to $\mu$ uniformly in the sequence of the concentric annuli $A_{n}:=\left\{\left|x_{n}\right| \leq|x| \leq\left|x_{n+1}\right|\right\}$, we construct two radial perturbations of $\mu$. Let $\mu_{-}(x):=\mu(x)-\delta|x|^{a}$ and $\mu_{+}(x):=\mu(x)+\delta|x|^{a}$ (for some $a>(p-d) /(p-1)$ ). It turns out that $\mu_{-}$(resp., $\left.\mu_{+}\right)$is a radial subsolution (resp., supersolution) of the equation $Q^{\prime}(u)=0$ near the origin, and therefore using the comparison principle in the annulus $A_{n}$ and (5.5), it follows that

$$
\lim _{r \rightarrow 0}\|v(x) / \mu(x)-1\|_{L^{\infty}(|x|=|r|)}=0 .
$$


Definition 5.3. Let $K$ be a compact set in $\Omega$. A positive solution of the equation $Q^{\prime}(u)=0$ in $\Omega \backslash K$ is said to be a positive solution of minimal growth in a neighborhood of infinity in $\Omega$, if for any compact set $K_{1}$ in $\Omega$, with a smooth boundary, satisfying $\operatorname{int}\left(K_{1}\right) \supset K$, and any positive supersolution $v \in C\left(\left(\Omega \backslash K_{1}\right) \cup \partial K_{1}\right)$ of the equation $Q^{\prime}(u)=0$ in $\Omega \backslash K_{1}$, the inequality $u \leq v$ on $\partial K_{1}$ implies that $u \leq v$ in $\Omega \backslash K_{1}$. A positive solution $u$ of the equation $Q^{\prime}(u)=0$ in $\Omega$, which has minimal growth in a neighborhood of infinity in $\Omega$ is called a global minimal solution of the equation $Q^{\prime}(u)=0$ in $\Omega$.

The following result is an extension to the $p$-Laplacian of the corresponding result of S. Agmon concerning positive solutions of real linear secondorder elliptic operators [1].

Theorem 5.4. Suppose that $1<p \leq d$, and $Q$ is nonnegative on $C_{0}^{\infty}(\Omega)$. Then for any $x_{0} \in \Omega$ the equation $Q^{\prime}(u)=0$ has (up to a multiple constant) a unique positive solution $v$ in $\Omega \backslash\left\{x_{0}\right\}$ of minimal growth in a neighborhood of infinity in $\Omega$.

Moreover, $v$ is either a global minimal solution of the equation $Q^{\prime}(u)=0$ in $\Omega$, or $v$ has a nonremovable singularity at $x_{0}$.

Proof. Take $x_{0} \in \Omega$, and consider an exhaustion $\left\{\Omega_{N}\right\}_{N=1}^{\infty}$ of $\Omega$ (as in the proof of Theorem 2.3). Fix $N \geq 1$, and denote $\Omega_{N, k}:=\Omega_{N} \backslash B\left(x_{0}, 1 / k\right)$.

Let $\left\{f_{k}\right\}$ be a sequence of nonzero nonnegative smooth functions such that for each $k \geq 2$, the function $f_{k}$ is supported in $B\left(x_{0}, 2 / k\right) \backslash B\left(x_{0}, 1 / k\right)$.

Recall that $\lambda_{1, p}\left(\Omega_{N, k}\right)>0$ for all $N, k \geq 1$. By Theorem 2.1, there exists a unique positive solution of the problem

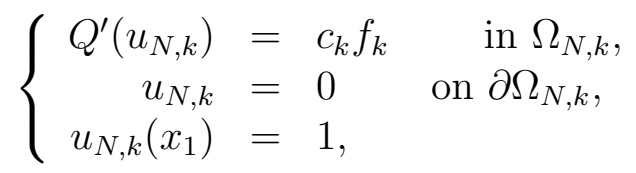

where $x_{1} \neq x_{0}$ is a fixed point in $\Omega_{1}$ and $c_{k}>0$. By Harnack's inequality and elliptic regularity, it follows that $\left\{u_{N, k}\right\}$ admits a subsequence which converges locally uniformly in $\Omega_{N} \backslash\left\{x_{0}\right\}$ to a positive solution $G_{N}\left(\cdot, x_{0}\right)$ of the equation $Q^{\prime}(u)=0$ in $\Omega_{N} \backslash\left\{x_{0}\right\}$. Moreover, $G_{N}\left(\cdot, x_{0}\right)=0$ on $\partial \Omega_{N}$, and $G_{N}\left(x_{1}, x_{0}\right)=1$.

Since $\lambda_{1, p}\left(\Omega_{N}\right)>0$, and it is the unique eigenvalue with a positive Dirichlet eigenfunction, it follows that $G_{N}\left(\cdot, x_{0}\right)$ has a nonremovable singularity at $x_{0}$. 
Recall that (5.3) holds true with $v(\cdot)=G_{N}\left(\cdot, x_{0}\right)$. It is convenient to normalize $G_{N}$ in the traditional way, so that

$$
\begin{array}{rlrl}
\lim _{x \rightarrow x_{0}} \frac{G_{N}\left(x, x_{0}\right)}{\left|x-x_{0}\right|^{\alpha(d, p)}} & =\frac{p-1}{d-p}\left|S^{d-1}\right|^{-1 /(p-1)} & & p<d, \\
\lim _{x \rightarrow x_{0}} \frac{G_{N}\left(x, x_{0}\right)}{-\log \left|x-x_{0}\right|} & =\left|S^{d-1}\right|^{-1 /(d-1)} & p=d,
\end{array}
$$

where $S^{d-1}$ is the unit sphere in $\mathbb{R}^{d}$. Using the comparison principle (Theorem 2.2), and (5.3), it follows that $G_{N}\left(\cdot, x_{0}\right)$ is the unique function with the above properties. Hence, $G_{N}\left(\cdot, x_{0}\right)$ might be called the (Dirichlet) positive $p$-Green function of the functional $Q$ in $\Omega_{N}$ with a pole at $x_{0}$.

By the comparison principle (Theorem 2.2) and (5.3), it follows that the sequence $\left\{G_{N}\left(\cdot, x_{0}\right)\right\}$ is nondecreasing as a function of $N$, and therefore it converges locally uniformly in $\Omega \backslash\left\{x_{0}\right\}$ either to a positive function $G\left(\cdot, x_{0}\right)$ or to infinity.

In the first case $G\left(\cdot, x_{0}\right)$ is a positive solution of the equation $Q^{\prime}(u)=0$ in $\Omega \backslash\left\{x_{0}\right\}$ and has the asymptotic behavior (5.3) near $x_{0}$. We call $G\left(\cdot, x_{0}\right)$ the minimal positive $p$-Green function of the functional $Q$ in $\Omega$ with a pole at $x_{0}$.

In the second case, we consider the normalized sequence

$$
v_{N}(x):=\frac{G_{N}\left(x, x_{0}\right)}{G_{N}\left(x_{1}, x_{0}\right)} \quad n=1,2, \ldots
$$

By Harnack's inequality and elliptic regularity, it follows that $\left\{v_{N}\right\}$ admits a subsequence which converges locally uniformly to a positive solution $v$ of the equation $Q^{\prime}(u)=0$ in $\Omega \backslash\left\{x_{0}\right\}$.

Assume that $v$ has a nonremovable singularity at $x_{0}$. Therefore, for each $N \geq 1$ we obtain by the comparison principle and (5.7) that

$$
G_{N}\left(x, x_{0}\right) \leq C v(x) \quad \forall x \in \Omega \backslash\left\{x_{0}\right\}
$$

for some $C>0$ independent of $N$. But this contradicts our assumption that $G_{N} \rightarrow \infty$ as $N \rightarrow \infty$.

Note that $G\left(\cdot, x_{0}\right)$ (in the first case) and $v$ (in the second case) are limits of a sequence of positive solutions that for $\delta>0$ are uniformly bounded on $\partial B\left(x_{0}, \delta\right)$, and take zero boundary condition on $\partial \Omega_{N}$. Therefore, by the comparison principle, $G$ and $v$ are positive solutions in $\Omega \backslash\left\{x_{0}\right\}$ of minimal 
growth in a neighborhood of infinity in $\Omega$. In particular, $v$ is a global minimal positive solution of the equation $Q^{\prime}(u)=0$ in $\Omega$.

Using again the comparison principle and (5.3), it follows that such a solution is unique.

The next theorem demonstrates that a global minimal positive solution of the equation $Q^{\prime}(u)=0$ in $\Omega$ is a ground state.

Theorem 5.5. Assume that $1<p \leq d$ and that $Q_{V} \geq 0$ on $C_{0}^{\infty}(\Omega)$. Then $Q_{V}$ is degenerately positive in $\Omega$ if and only if the equation $Q^{\prime}(u)=0$ admits a global minimal positive solution in $\Omega$.

Proof. Assume that $Q_{V}$ is strictly positive and assume that there exists a global minimal positive solution $v$ of the equation $Q^{\prime}(u)=0$ in $\Omega$. By Proposition 4.4, there exists a nonzero nonnegative function $V_{1} \in C_{0}^{\infty}(\Omega)$ with supp $V_{1} \subset B\left(x_{0}, \delta\right)$ for some $\delta>0$, such that $Q_{V-V_{1}}$ is strictly positive in $\Omega$. Therefore, in light of Theorem 2.3 , there exists a positive solution $v_{1}$ of the equation $Q_{V-V_{1}}^{\prime}(u)=0$ in $\Omega$.

Clearly, $v_{1}$ is a positive supersolution of the equation $Q_{V}^{\prime}(u)=0$ in $\Omega$ which is not a solution. On the other hand, $v$ is a positive solution of the equation $Q_{V}^{\prime}(u)=0$ in $\Omega$ which has minimal growth in a neighborhood of infinity in $\Omega$. Therefore, there exists $\varepsilon>0$ such that $\varepsilon v \leq v_{1}$ in $\Omega$. Define

$$
\varepsilon_{0}:=\max \left\{\varepsilon>0 \mid \varepsilon v \leq v_{1} \text { in } \Omega\right\} .
$$

Clearly $\varepsilon_{0} v \supsetneqq v_{1}$ in $\Omega$. Consequently, there exist $\delta_{1}, \delta_{2}>0$ and $x_{1} \in \Omega$ such that

$$
\left(1+\delta_{1}\right) \varepsilon_{0} v(x) \leq v_{1}(x) \quad x \in B\left(x_{1}, \delta_{2}\right) .
$$

Hence, by the definition of minimal growth, we have

$$
\left(1+\delta_{1}\right) \varepsilon_{0} v(x) \leq v_{1}(x) \quad x \in \Omega \backslash B\left(x_{1}, \delta_{2}\right),
$$

and thus $\left(1+\delta_{1}\right) \varepsilon_{0} v \leq v_{1}$ in $\Omega$, which is a contradiction to the definition of $\varepsilon_{0}$.

Assume that $Q$ admits a positive minimal $p$-Green function $G\left(\cdot, x_{0}\right)$ in $\Omega$. We need to prove that $Q$ is strictly positive.

Consider an exhaustion $\left\{\Omega_{N}\right\}_{N=1}^{\infty}$ of $\Omega$ such that $x_{0} \in \Omega_{1}$ and $x_{1} \in \Omega \backslash \Omega_{1}$. Fix a nonzero nonnegative function $f \in C_{0}^{\infty}\left(\Omega_{1}\right)$. By Theorem 2.1, there exists a unique positive solution of the Dirichlet problem

$$
\left\{\begin{aligned}
Q^{\prime}\left(u_{N}\right)=f & \text { in } \Omega_{N} \\
u_{N}=0 & \text { on } \partial \Omega_{N}
\end{aligned}\right.
$$


By the comparison principle (Theorem 2.2), $\left\{u_{N}\right\}$ is an increasing sequence. Suppose that $\left\{u_{N}\left(x_{1}\right)\right\}$ is bounded. Then $u_{N} \rightarrow u$, where $u$ satisfies the equation $Q^{\prime}(u)=f \gtreqless 0$ in $\Omega$. Since $u$ is a positive supersolution of the equation $Q^{\prime}(u)=0$ in $\Omega$ which is not a solution, Theorem 1.6 implies that $Q$ is strictly positive.

Suppose that $u_{N}\left(x_{1}\right) \rightarrow \infty$. Then $v_{N}(x):=u_{N}(x) / u_{N}\left(x_{1}\right)$ solves the problem

$$
\left\{\begin{aligned}
Q^{\prime}\left(v_{N}\right) & =\frac{f(x)}{u_{N}\left(x_{1}\right)^{p-1}} & & \text { in } \Omega_{N}, \\
v_{N} & =0 & & \text { on } \partial \Omega_{N}, \\
v_{N}\left(x_{1}\right) & =1 . & &
\end{aligned}\right.
$$

By Harnack's inequality, and the comparison principle,

$$
v_{N} \asymp G_{N}\left(\cdot, x_{0}\right) \quad \text { in } \Omega_{N} \backslash \Omega_{1} .
$$

By a standard elliptic argument, we may extract a subsequence of $\left\{v_{N}\right\}$ that converges to a positive supersolution $v$ of the equation $Q^{\prime}(u)=0$ in $\Omega$. Recall that $G_{N}\left(\cdot, x_{0}\right) \rightarrow G\left(\cdot, x_{0}\right)$. Hence, (5.10) implies that $v \asymp G\left(\cdot, x_{0}\right)$ in $\Omega \backslash \Omega_{1}$, and in particular, $v$ is a positive solution in $\Omega \backslash \Omega_{1}$ of minimal growth in a neighborhood of infinity in $\Omega$. Note that $v \neq c G\left(\cdot, x_{0}\right)$ since $v$ is not singular at $x_{0}$. Since the equation $Q^{\prime}(u)=0$ does not admit a global minimal solution in $\Omega$, it follows that $v$ satisfies $Q^{\prime}(u) \gtreqless 0$ in $\Omega$, and by Theorem 1.6, $Q$ is strictly positive in $\Omega$.

\section{Open problems}

We conclude the paper with a number of open problems suggested by the above results which are left for future investigation. All these questions are already resolved when $p=2$.

The first problem (Problem 6.1) deals with the weakly lower semicontinuity and convexity of the functional $Q$. As was shown in Proposition 3.5, $Q$ is weakly lower semicontinuous in a limited sense, a property which is closely related to convexity. However, $Q$ is not necessarily convex even if $Q \geq 0$ on $C_{0}^{\infty}(\Omega)$ : for $p>2$ see the elementary one-dimensional counterexample at the end of [9], and also the proof of Theorem 7 in [17], for $p<2$ see [14], Example 2. Note also that Proposition 3.5 does not assert that the domain $D$ defined by (3.15) is weakly closed. 
Problem 6.1. Does $Q$ have a natural extension to a weakly closed set where it is weakly lower semicontinuous? Under what conditions a nonnegative functional $Q$ of the form (1.1) is convex?

Clearly $Q$ is convex when $V \geq 0$ or $p=2$. The convexity of $Q$ gives rise to an energy space for the form $Q$ that would generalize the space $\mathcal{D}^{1, p}$, similarly to the known case $p=2$ [32]. Indeed, if $Q$ is a nonnegative convex functional on $C_{0}^{\infty}(\Omega)$, then it follows that $Q^{\frac{1}{p}}$ is a norm on $C_{0}^{\infty}(\Omega)$. Moreover, by Theorem 1.6, if $Q$ has a weighted spectral gap in $\Omega$, then the completion of $C_{0}^{\infty}(\Omega)$ with respect to this norm is continuously imbedded into $L_{\text {loc }}^{p}(\Omega)$. On the other hand, if $Q$ has a ground state $v$ in $\Omega$, then $v$ belongs to the equivalence class of 0 in this completion, and there is no continuous imbedding of the completion even into $\mathcal{D}^{\prime}(\Omega)$. However, due to (1.7), the completion of $C_{0}^{\infty}(\Omega)$ with respect to the norm induced by the right hand side of (1.7) is continuously imbedded into $L_{\mathrm{loc}}^{p}(\Omega)$.

Problem 6.2. Do the results of this paper extend to quasilinear functionals of the form

$$
Q^{A}(u):=\int_{\Omega}\left(|A(x) \nabla u|^{p}+V|u|^{q}\right) \mathrm{d} x,
$$

where $A$ is a strictly positive definite matrix and $1<p \leq q<\infty$ ?

We note that Picone-type identity for the case $A(x)=a(x) I$ was established in [22].

Problem 6.3. Generalize the results of Section 5 to the case $d<p<\infty$.

Problem 6.4. Let $\Omega_{1} \varsubsetneqq \Omega$ be domains in $\mathbb{R}^{d}$. Suppose that $Q$ is strictly positive in $\Omega_{1}$. Show that there exists an open domain $\Omega_{1} \varsubsetneqq \Omega_{2} \subset \Omega$ such that $Q_{V}$ is strictly positive in $\Omega_{2}$.

Problem 6.4 was studied in [31] under the assumption $p=2$, and stronger statements were proved.

Problem 6.5. Let $\Omega=\mathbb{R}^{d}$ and assume that $Q=Q_{V_{j}}$ are strictly positive in $\Omega$, for $j=1,2$. For $y \in \mathbb{R}^{d}$ denote $V_{y}(x):=V_{1}(x)+V_{2}(x-y)$. Show that under suitable decay conditions on $V_{j}$, there exists $R>0$ such that for every $y \in \mathbb{R}^{d} \backslash B_{R}(0)$ the functional $Q_{V_{y}}$ is strictly positive in $\Omega$.

This phenomenon has been proved for $p=2$ in [24, 26, 36] for Schrödinger operators and in [29] for the non-selfadjoint case. 


\section{A Appendix: Energy inequality}

The following inequality, established for $p \geq 2$, estimates the functional $Q$ from below by an expression that leads to an alternative proof of Lemma 3.2 for the case $p \geq 2$.

Lemma A.1. Assume that $p \geq 2$. Let $v \in C^{1}(\Omega)$ be a positive solution of the equation $Q^{\prime}(u)=0$ in $\Omega$, and let $u \in W_{\text {loc }}^{1, p}(\Omega), u \geq 0$, supp $u \Subset \Omega$. Then

$$
Q(u) \begin{cases}\geq Q_{1}(u)+Q_{2}(u) & \text { if } p>2, \\ =Q_{1}(u) & \text { if } p=2 .\end{cases}
$$

where

$Q_{1}(u):=\frac{2}{p} \int_{\Omega}|\nabla v|^{p-2} v^{2}\left|\nabla\left[\left(\frac{u}{v}\right)^{\frac{p}{2}}\right]\right|^{2} \mathrm{~d} x, \quad$ and $\quad Q_{2}(u):=\int_{\{\nabla v=0\}}|\nabla u|^{p} \mathrm{~d} x$.

Proof. Since $p \geq 2$, the obvious inequality $t^{p}+(p-1)-p t \geq(p-1)(t-1)^{2}$ implies

$$
L_{1}(u, v) \geq(p-1)\left(\frac{|\nabla u|}{u}-\frac{|\nabla v|}{v}\right)^{2}\left(\frac{u}{v}\right)^{p}|\nabla v|^{p-2} v^{2},
$$

where $L_{1}$ is defined by (2.4). We use the identity

$$
\left|\nabla\left[\left(\frac{u}{v}\right)^{\frac{p}{2}}\right]\right|^{2}=\left(\frac{p}{2}\right)^{2}\left(\frac{u}{v}\right)^{p}\left|\frac{\nabla u}{u}-\frac{\nabla v}{v}\right|^{2} .
$$

Substitution of (A.3) into (A.2) and using the identity

$$
\left(\frac{|\nabla u|}{u}-\frac{|\nabla v|}{v}\right)^{2}-\left|\frac{\nabla u}{u}-\frac{\nabla v}{v}\right|^{2}=2 \frac{\nabla u \cdot \nabla v-|\nabla u||\nabla v|}{u v}
$$

gives

$$
\begin{gathered}
L_{1}(u, v) \geq(p-1)\left(\frac{2}{p}\right)^{2}|\nabla v|^{p-2} v^{2}\left|\nabla\left[\left(\frac{u}{v}\right)^{\frac{p}{2}}\right]\right|^{2}+ \\
2(p-1)(\nabla u \cdot \nabla v-|\nabla u||\nabla v|)\left(\frac{u}{v}\right)^{p-1}|\nabla v|^{p-2},
\end{gathered}
$$

which is the same as 


$$
L_{1}(u, v) \geq(p-1)\left(\frac{2}{p}\right)^{2}|\nabla v|^{p-2} v^{2}\left|\nabla\left[\left(\frac{u}{v}\right)^{\frac{p}{2}}\right]\right|^{2}-2 \frac{p-1}{p} L_{2}(u, v),
$$

where $L_{2}$ is defined by (2.5). Since $1 \geq 2 /\left(p^{2}-p\right)$ for $p \geq 2$, (A.5) implies that

$$
L(u, v) \geq \frac{2}{p}|\nabla v|^{p-2} v^{2}\left|\nabla\left[\left(\frac{u}{v}\right)^{\frac{p}{2}}\right]\right|^{2} .
$$

Note that for $p=2$ we have $L(u, v)=v^{2}\left|\nabla\left(\frac{u}{v}\right)\right|^{2}$ (see [32, Lemma 2.4]).

Moreover, on the critical set $\{x \in \Omega|| \nabla v(x) \mid=0\}$, we have $L(u, v)=$ $|\nabla u|^{p}$. Therefore for $p>2$ we have

$$
L(u, v) \geq \frac{2}{p}|\nabla v|^{p-2} v^{2}\left|\nabla\left[\left(\frac{u}{v}\right)^{\frac{p}{2}}\right]\right|^{2}+\mathbf{1}_{\{|\nabla v|=0\}}|\nabla u|^{p} .
$$

Integrating the latter inequality over $\Omega$, we arrive at (A.1).

Remark A.2. 1. Note that (A.1) is based on the pointwise inequality (A.6).

2. Note that for $p=2$ we have $Q(u)=Q_{1}(u)$ (see for example [32, Lemma 2.4]). The set of all critical points of a positive solution of the linear equation $-\Delta u+V u=0$ in $\Omega$ has studied in [20].

Alternative proof of Lemma 3.2. Assume that $p \geq 2$. By (A.1), if $Q\left(u_{k}\right) \rightarrow 0$, then $Q_{1}\left(u_{k}\right) \rightarrow 0$ and $Q_{2}\left(u_{k}\right) \rightarrow 0$. Since $c_{B}=0$, there exists a sequence $u_{k} \in C_{0}^{\infty}(\Omega), u_{k} \geq 0$, such that $\int_{B} u_{k}^{p}=1$ and $Q\left(u_{k}\right) \rightarrow 0$. Repeating the first two steps of the proof of Lemma 3.2, we deduce that $\left\{u_{k}\right\}$ is bounded in $W^{1, p}(\omega)$ for every $\omega \Subset \Omega, \omega \supset B$.

Consider now a weakly convergent renamed subsequence $u_{k} \rightarrow u$ in $W^{1, p}(\omega)$. Let

$$
Q_{1}^{\omega}(w):=\frac{2}{p} \int_{\omega}|\nabla v|^{p-2} v^{2}\left|\nabla\left[\left(\frac{w}{v}\right)^{\frac{p}{2}}\right]\right|^{2} \mathrm{~d} x, \quad \text { and } Q_{2}^{\omega}(w):=\int_{\{\nabla v=0\} \cap \omega}|\nabla w|^{p} \mathrm{~d} x \text {. }
$$

Since $Q_{1}^{\omega}$ and $Q_{2}^{\omega}$ are continuous convex functionals on $W^{1, p}(\omega)$, they are weakly lower semicontinuous, and therefore,

$$
Q_{1}^{\omega}(u) \leq \lim _{k \rightarrow \infty} Q_{1}^{\omega}\left(u_{k}\right)=0 \quad \text { and } \quad Q_{2}^{\omega}(u) \leq \lim _{k \rightarrow \infty} Q_{2}^{\omega}\left(u_{k}\right)=0
$$


Consequently, $\nabla\left[(u / v)^{\frac{p}{2}}\right]=0$ almost everywhere in $\omega \backslash\{\nabla v=0\}$ and $\nabla u=0$ in $\omega \cap\{\nabla v=0\}$. Note that if $\nabla u(x)=0$ and $\nabla v(x)=0$, then $\nabla\left[(u(x) / v(x))^{\frac{p}{2}}\right]=0$. Thus, $\nabla\left[(u / v)^{\frac{p}{2}}\right]=0$ a.e. in $\omega$, and since it holds for every $\omega$ containing $B$, it follows that $u / v=$ constant a.e. in $\omega$. By the compact Sobolev imbedding on $B, \int_{B} u^{p} \mathrm{~d} x=\lim \int_{B} u_{k}^{p} \mathrm{~d} x=1$, and therefore, $u=c v$, where $c^{-p}=\int_{B} v^{p} \mathrm{~d} x$. Note that any subsequence of $\left\{u_{k}\right\}$ has a subsequence converging to $c v$ with the same $c$. From the compactness of the local Sobolev imbedding, it follows that $u_{k} \rightarrow c v$ in $L_{\mathrm{loc}}^{p}(\Omega)$. In other words, $\left\{u_{k}\right\}$ is a null sequence.

\section{Acknowledgments}

The authors are grateful to L. Véron for kindly providing the authors the proof of Lemma 5.1, a contribution which has substantially improved the coherence of the paper. The authors wish to thank V. Liskevich and V. Moroz for valuable discussions, in particular for V. Moroz' comment on the convexity counterexamples in [9], 17] and [14]. Part of this research was done while K. T. was visiting the Technion and the Hebrew University, and Y. P. was visiting the Forschungsinsitut für Mathematik, ETH. The authors would like to thank these institutes for the kind hospitality. The work of Y. P. was partially supported by the RTN network "Nonlinear Partial Differential Equations Describing Front Propagation and Other Singular Phenomena", HPRN-CT-2002-00274, and the Fund for the Promotion of Research at the Technion. The work of K. T. was partially supported by the Swedish Research Council.

\section{References}

[1] S. Agmon, On positivity and decay of solutions of second order elliptic equations on Riemannian manifolds, in "Methods of Functional Analysis and Theory of Elliptic Equations" (Naples, 1982), 19-52, Liguori, Naples, 1983.

[2] W. Allegretto, and Y. X. Huang, A Picone's identity for the p-Laplacian and applications, Nonlinear Anal. 32 (1998), 819-830.

[3] W. Allegretto, and Y. X. Huang, Principal eigenvalues and Sturm comparison via Picone's identity, J. Differential Equations 156 (1999), 427438. 
[4] G. Barbatis, S. Filippas, and A. Tertikas, A unified approach to improved $L^{p}$ Hardy inequalities with best constants, Trans. Amer. Math. Soc. 356 (2004), 2169-2196.

[5] M.-F. Bidaut-Véron, R. Borghol, and L. Véron, Boundary Harnack inequality and a priori estimates of singular solutions of quasilinear elliptic equations, to appear in Calc. Var. Partial Differential Equations.

[6] H. Brezis, and E. H. Lieb, Sobolev inequalities with remainder terms. $J$. Funct. Anal. 62 (1985), 73-86.

[7] H. Brezis, and M. Marcus, Hardy's inequalities revisited, Dedicated to Ennio De Giorgi, Ann. Scuola Norm. Sup. Pisa Cl. Sci. (4) 25 (1997), 217-237.

[8] H. L. Cycon, R. G. Froese, W. Kirsch, and B. Simon, "Schrödinger Operators with Applications to Quantum Mechanics and Global Geometry", Texts and Monographs in Physics, Springer Verlag, Berlin, 1987.

[9] M. del Pino, M. Elgueta,and R. Manasevich, A homotopic deformation along $p$ of a Leray-Schauder degree result and existence for $\left(\left|u^{\prime}\right|^{p-2} u^{\prime}\right)^{\prime}+$ $f(t, u)=0, u(0)=u(T)=0, p>1, J$. Differential Equations 80 (1989), 1-13.

[10] E. DiBenedetto, $C^{1+\alpha}$ local regularity of weak solutions of degenerate elliptic equations, Nonlinear Anal. 7 (1983), 827-850.

[11] P. Drábek, P. Girg, P. Takáč, and M. Ulm, The Fredholm alternative for the $p$-Laplacian: bifurcation from infinity, existence and multiplicity, Indiana Univ. Math. J. 53 (2004), 433-482.

[12] P. Drábek, A. Kufner, and F. Nicolosi, "Quasilinear Elliptic Equations with Degenerations and Singularities", de Gruyter Series in Nonlinear Analysis and Applications 5, Walter de Gruyter \& Co., Berlin, 1997.

[13] S. Filippas, and A. Tertikas, Optimizing improved Hardy inequalities, J. Funct. Anal. 192 (2002), 186-233; Corrigendum, J. Funct. Anal. 255 (2008), 2095. 
[14] J. Fleckinger-Pellé, J. Hernández, P. Takáč, and F. de Thélin, Uniqueness and positivity for solutions of equations with the $p$-Laplacian, Proceedings of the Conference on Reaction-Diffusion Equations (Trieste, 1995), 141-155, Lecture Notes in Pure and Applied Math. Vol. 194, Marcel Dekker, New York, 1998.

[15] J. Fleckinger-Pellé, R. F. Manásevich, N. M. Stavrakakis, and F. de Thélin, Principal eigenvalues for some quasilinear elliptic equations on $\mathbb{R}^{N}$, Adv. Differential Equations 2 (1997), 981-1003.

[16] J. Fleckinger-Pellé, J-P. Gossez, and F. de Thélin, Antimaximum principle in $\mathbb{R}^{N}$ : local versus global, J. Differential Equations 196 (2004), 119-133.

[17] J. García-Melián, and J. Sabina de Lis, Maximum and comparison principles for operators involving the $p$-Laplacian, J. Math. Anal. Appl. 218 (1998), 49-65.

[18] D. Gilbarg, and J. Serrin, On isolated singularities of solutions of second order elliptic differential equations, J. Analyse Math. 4 (1955/56), 309340 .

[19] M. Guedda, and L. Véron, Local and global properties of solutions of quasilinear elliptic equations, J. Differential Equations 76 (1988), 159189.

[20] R. Hardt, M. Hoffmann-Ostenhof, T. Hoffmann-Ostenhof, and N. Nadirashvili, Critical sets of solutions to elliptic equations, J. Differential Geom. 51 (1999), 359-373.

[21] J. Heinonen, T. Kilpeläinen, and O. Martio, "Nonlinear Potential Theory of Degenerate Elliptic Equations", Oxford Mathematical Monographs, Oxford University Press, New York, 1993.

[22] J. Jaroš, K. Takaŝi, and N. Yoshida, Picone-type inequalities for halflinear elliptic equations and their applications, Adv. Math. Sci. Appl. 12 (2002), 709-724.

[23] S. Kichenassamy, and L. Véron, Singular solutions of the p-Laplace equation, Math. Ann. 275 (1986), 599-615. 
[24] M. Klaus, and B. Simon, Binding of Schrödinger particles through conspiracy of potential wells, Ann. Inst. Henri Poincaré, Sect. A: Physique théorique 30 (1979), 83-87.

[25] M. Marcus, and I. Shafrir, An eigenvalue problem related to Hardy's $L^{p}$ inequality, Ann. Scuola Norm. Sup. Pisa Cl. Sci. (4) 29 (2000), 581-604.

[26] Yu. N. Ovchinnikov, and I. M. Sigal, Number of bound states of threebody systems and Efimov's effect, Ann. Phys. 123 (1979), 274-295.

[27] Y. Pinchover, On positive solutions of second-order elliptic equations, stability results, and classification, Duke Math. J. 57 (1988), 955-980.

[28] Y. Pinchover, On criticality and ground states of second order elliptic equations, II, J. Differential Equations 87 (1990), 353-364.

[29] Y. Pinchover, On the localization of binding for Schrödinger operators and its extension to elliptic operators, J. Anal. Math. 66 (1995), 57-83.

[30] Y. Pinchover, On principal eigenvalues for indefinite-weight elliptic problems, in: Spectral and Scattering Theory (Newark, DE, 1997), 77-87, Plenum, New York, 1998.

[31] Y. Pinchover, and K. Tintarev, Existence of minimizers for Schrödinger operators under domain perturbations with application to Hardy's inequality, Indiana Univ. Math. J. 54 (2005), 1061-1074.

[32] Y. Pinchover, and K. Tintarev, Ground state alternative for singular Schrödinger operators, to appear in J. Functional Analysis, 230 (2006), 65-77.

[33] A. Poliakovsky, and I. Shafrir, Uniqueness of positive solutions for singular problems involving the $p$-Laplacian, Proc. Amer. Math. Soc. 133 (2005), 2549-2557.

[34] J. Serrin, Local behavior of solutions of quasi-linear equations, Acta Math. 111 (1964), 247-302.

[35] J. Serrin, Isolated singularities of solutions of quasi-linear equations, Acta Math. 113 (1965), 219-240. 
[36] B. Simon, Brownian motion, $L^{p}$ properties of Schrödinger operators and the localization of binding, J. Funct. Anal. 35 (1980), 215-229.

[37] P. Tolksdorf, Regularity for a more general class of quasilinear elliptic equations, J. Differential Equations 51 (1984), 126-150.

[38] L. Véron, "Singularities of Solutions of Second Order Quasilinear Equations", Pitman Research Notes in Mathematics Series, 353. Longman, Harlow, 1996.

[39] W. P. Ziemer, "Weakly Differentiable Functions. Sobolev Spaces and Functions of Bounded Variation", Graduate Texts in Mathematics, 120. Springer-Verlag, New York, 1989. 